\title{
Definición del concepto de consumo intensivo de alcohol adolescente (binge drinking)
}

\section{Definition of adolescent binge drinking}

\author{
María Parada*; Montserrat Corral*; Francisco \\ CaAmaño-Isorna*; Nayara Mota*; Alberto Crego*; \\ Socorro Rodríguez Holguín*; Fernando Cadaveira*
}

\author{
Departamento de Psicología Clínica y Psicobiología, Universidad de \\ Santiago de Compostela, España. \\ ** CIBER de Epidemiología y Salud Pública (CIBERESP), Departamento \\ de Medicina Preventiva, Universidad de Santiago de Compostela, \\ España.
}

\section{RESUMEN}

Objetivos: Delimitar el concepto de consumo intensivo de alcohol adolescente (binge drinking) con el fin de definir un criterio operativo del término.

Metodología: Se ha realizado una revisión bibliográfica en las bases de datos Medline y Psyclit en el periodo 1980-2009. Mediante el filtro "(binge drinking OR heavy-episodic-drinking) AND (adolescence OR university-students $O R$ college-students)" se seleccionaron 80 artículos para su revisión.

Resultados: Los estudios epidemiológicos que han informado sobre la prevalencia de este patrón de consumo muestran falta de consenso a la hora de operativizar el término. Se han propuesto distintos criterios en cuanto al número de unidades de bebida estándar (UBEs) consumidas por ocasión, la frecuencia de los episodios y la duración de los mismos. Conclusiones: Una definición adecuada del patrón de consumo intensivo de alcohol debe integrar las variables cantidad y frecuencia y, además, tener en cuenta las consecuencias negativas asociadas al mismo. El criterio más comúnmente aceptado por la comunidad científica internacional es el consumo de 5 ó más UBEs - 4 ó más para mujeres- en una única ocasión, al menos una vez en las dos últimas semanas. Sin embargo, aspectos como las diferencias entre paises en los gramos de alcohol de las UBEs dificultan el establecimiento de una definición internacional del término. Esto hace necesario adaptar este criterio al pais donde se realice el estudio.

Palabras clave: consumo intensivo de alcohol, adolescencia, estudiantes universitarios, revisión, epidemiología.
Enviar correspondencia a:

María Parada Iglesias. Departamento de Psicología Clínica y

Psicobiología, Facultad de Psicología, Campus Sur s/n, 15782

Santiago de Compostela, España.

Teléfono: 981563100 (ext. 13766). Fax: 981528072

Correo electrónico: maria.parada@usc.es

recibido: Mavo 2010 aceptado: Octubre 2010

\section{ABSTRACT}

Background: Review of the concept of adolescent binge drinking in order to propose an operational definition.

Methods: We conducted a literature review in the databases MEDLINE and PSYCLIT for the period 1980 to 2009. Through the filter "(binge drinking OR heavy-episodic-drinking) AND (adolescence OR universitystudents OR college-students)" 80 articles were selected for review.

Results: Epidemiological studies that have reported the prevalence of binge drinking show a lack of consensus regarding the operational definition. Alternative approaches have been proposed in relation to the number of standard drink units (SDUs) consumed per occasion, frequency of episodes and their duration.

Conclusions: A proper definition of the pattern of intensive alcohol consumption should integrate the quantity and frequency variables, and also take into account the negative consequences associated with it. The criterion most widely accepted by the international scientific community is the consumption of 5 or more SDUs - 4 or more for women - on a single occasion at least once in the last two weeks. However, aspects such as differences in the grams of alcohol of SDUs hinder the establishment of an international definition of the term, making it necessary to adapt this approach to the country in which the study is being carried out.

Keywords: binge drinking, adolescence, college or university students, review, epidemiology. 


\section{INTRODUCCIÓN}

A unque el consumo de alcohol ha disminuido en los últimos años, ésta sigue siendo la droga psicoactiva más consumida en nuestro país, de la que más se abusa y la que más problemas sociales y sanitarios causa. El consumo de alcohol se relaciona con accidentes de tráfico, violencia doméstica, maltrato infantil, problemas laborales, urgencias e ingresos hospitalarios, y en general con mayor morbilidad y mortalidad por todas las causas ${ }^{1,2}$.

En España el consumo de alcohol se ha asociado tradicionalmente a la población adulta y se ha caracterizado por la regularidad de su uso, y su vinculación a la dieta y a los acontecimientos sociales. Sin embargo, en los últimos veinte años se han experimentado cambios muy importantes tanto en las cantidades ingeridas, como en los patrones y en el significado del consumo ${ }^{3,4}$.

Así, en los últimos informes del Observatorio Español sobre Drogas ${ }^{1,5}$ se ha destacado un patrón de consumo intermitente, en forma de atracones, concentrado en sesiones de pocas horas, asociado principalmente a las noches de fin de semana y que se realiza con grupos de iguales. Este patrón se caracteriza por picos de incidencia entre los jóvenes, igualación del consumo entre sexos, rejuvenecimiento del perfil de los bebedores abusivos y escasa percepción de riesgo. Es lo que la literatura anglosajona denomina binge drinking o heavy episodic drinking y que en este trabajo llamaremos consumo intensivo de alcohol (CIA).

La última encuesta domiciliaria sobre alcohol y drogas en España ${ }^{6}$ ha puesto de manifiesto que el $18.0 \%$ de la población de 15 a 34 años refiere haber ingerido 5 ó más bebidas alcohólicas en una misma ocasión (entendiendo por ocasión el tomar varias copas o vasos seguidos o en un par de horas) durante los últimos 30 días. La media de edad del primer consumo de alcohol es de 16.8 años.

Aún cuando el CIA no responde a los criterios habituales de consumo de riesgo (ni en cantidad ingerida, ni en frecuencia), diversos autores han puesto de manifiesto que sus consecuencias sociales y sanitarias son tan, o incluso más, importantes que las derivadas del consumo de riesgo regular-10. Además se ha sugerido que el $\mathrm{ClA}$ es un factor de riesgo para el desarrollo posterior de abuso/dependencia de alcohol ${ }^{11-13}$.

El estudio de los factores de riesgo y consecuencias sociosanitarias de este patrón de consumo en nuestro país requiere de una delimitación precisa de sus características. Desde el ámbito de la prevención y promoción de la salud pública, se ha propuesto la siguiente definición operativa del término $\mathrm{CIA}$ : consumo de 60 ó más gramos de alcohol en varones y de 40 o más gramos en mujeres, concentrado en una sesión de consumo (habitualmente 4-6 horas), durante la que se mantiene un cierto nivel de intoxicación (alcoholemia no inferior a $0,8 \mathrm{~g} / \mathrm{L})^{14}$. Sin embargo, desde el ámbito de la investigación clínica este término todavía no presenta un claro consenso.
El objetivo del presente trabajo es revisar el concepto de consumo intensivo de alcohol con el fin de encontrar un criterio operativo del término.

\section{MATERIAL Y MÉTODOS}

Se ha realizado una revisión bibliográfica en las bases de datos MEDLINE y PSYCLIT en el período 19802009. Se revisaron todos los artículos seleccionados a partir del filtro "(binge-drinking OR heavy-episodicdrinking) AND (adolescence OR university-students OR college-students)". También se revisaron los resúmenes de los artículos pertinentes incluidos en las referencias bibliográficas. En MEDLINE se encontraron 858 artículos y en PSYCLIT se encontraron 3.387. Dado el excesivo número que proporcionaba PSYCLIT se seleccionaron aquellos que contenían los términos del filtro como palabras clave, reduciéndose el número a 645 . Finalmente, se seleccionaron 80 artículos para la revisión. Los criterios empleados para su selección fueron: a) que abordaran de forma especifica la definición del concepto de $\mathrm{CIA}$, y b) que fueran representativos en términos geográficos y muestrales.

\section{RESULTADOS}

Los diversos estudios epidemiológicos que han informado sobre la prevalencia del patrón de consumo intensivo de alcohol muestran una gran variabilidad en el porcentaje de jóvenes que se podrian clasificar como consumidores intensivos de alcohol (entre el $7 \%$ y el $40 \%)^{15-18}$. Parte de esta variabilidad responde a la falta de consenso a la hora de operativizar el término $\mathrm{CIA}$ y a las diferencias de etanol puro que contiene una unidad de bebida estándar (UBE) en cada país. Así, los estudios han empleado distintos puntos de corte en cuanto al número de UBEs consumidas por ocasión ( 5 bebidas alcohólicas, 6 bebidas alcohólicas, etc...) y diferentes intervalos de frecuencia (última semana, 15 días, 30 dias, etc...) en los que se producen los episodios de consumo intensivo. La Tabla 1 resume los estudios analizados de modo que reflejen los criterios mayoritariamente empleados.

\section{DISCUSIÓN}

Una definición adecuada del CIA debe integrar las variables cantidad y frecuencia $y$, además, tener en cuenta las consecuencias negativas asociadas al consumo de alcoho ${ }^{19}$. Las numerosas definiciones propuestas hasta el momento han tenido en cuenta estas variables, si bien no siempre de forma integrada.

\section{Cantidad de alcohol ingerida}

Desde el estudio epidemiológico realizado en los años cincuenta por Strauss y Bacon en población 
Tabla 1. Estudios de consumo intensivo de alcohol en adolescentes y/o universitarios.

\begin{tabular}{|c|c|c|c|c|}
\hline ESTUDIO & PAÍS & OBJETIVO & SUJETOS & CRITERIO DE CIA \\
\hline O'Malley et al., $1984^{23}$ & EEUU & $\begin{array}{l}\text { Estimar la prevalencia del consumo de } \\
\text { alcohol y otras drogas. }\end{array}$ & $\begin{array}{l}\text { Estudiantes universitarios } \\
\text { entre } 18 \text { y } 24 \text { años. }\end{array}$ & $\begin{array}{l}\text { Consumo de } 5 \text { ó más bebidas alcohólicas en una única } \\
\text { ocasión en las dos últimas semanas. }\end{array}$ \\
\hline Wechsler et al., $1995^{25}$ & EEUU & $\begin{array}{l}\text { Estimar la prevalencia de CIA en población } \\
\text { universitaria. }\end{array}$ & $\begin{array}{l}17.592 \text { estudiantes de } 140 \\
\text { campus. }\end{array}$ & $\begin{array}{l}\text { Consumo de } 5 \text { ó más bebidas ( } 4 \text { ó más para mujeres) en } \\
\text { una única ocasión en la dos últimas semanas. }\end{array}$ \\
\hline Ariza et al. $2000^{66}$ & España & $\begin{array}{l}\text { Estudio de los factores asociados al } \\
\text { consumo problemático de alcohol en } \\
\text { adolescentes. }\end{array}$ & $\begin{array}{l}1.345 \text { hombres y } 795 \\
\text { mujeres (edad media de } \\
15,4 \text { años). }\end{array}$ & $\begin{array}{l}\text { Consumo de } 4 \text { ó más bebidas en una única ocasión al } \\
\text { menos una vez en su vida. }\end{array}$ \\
\hline Vik et al., $2000^{55}$ & EEUU & $\begin{array}{l}\text { Comparar la clasificación de CIA basada en } \\
\text { las } 2 \text { últimas semanas con una basada en } \\
\text { los últimos } 3 \text { meses. }\end{array}$ & $\begin{array}{l}40 \text { hombres y } 72 \text { mujeres } \\
\text { estudiantes universitarios } \\
\text { (edad media de 22,13 } \\
\text { años) }\end{array}$ & $\begin{array}{l}\text { Consumo de } 5 \text { ó más bebidas ( } 4 \text { ó más para mujeres) por } \\
\text { ocasión. }\end{array}$ \\
\hline Baer et al., $2001^{43}$ & EEUU & $\begin{array}{l}\text { Valorar si la prevención ayuda a reducir } \\
\text { a largo plazo el consumo intensivo de } \\
\text { alcohol. }\end{array}$ & $\begin{array}{l}4.000 \text { estudiantes de } 19 \\
\text { años. }\end{array}$ & $\begin{array}{l}\text { Consumo de } 6 \text { bebidas ( } 5 \text { para mujeres) en una única } \\
\text { ocasión al menos una vez en el último mes o experimentar } \\
3 \text { ó más consecuencias negativas relacionadas con el alco- } \\
\text { hol de } 3 \text { a } 5 \text { veces en los últimos tres años. }\end{array}$ \\
\hline
\end{tabular}

\begin{tabular}{|c|c|c|c|c|}
\hline $\begin{array}{l}\text { Christiansen et al., } \\
2002^{30}\end{array}$ & EEUU & $\begin{array}{l}\text { Estimar la prevalencia de estudiantes uni- } \\
\text { versitarios que consumen solos. }\end{array}$ & $\begin{array}{l}121 \text { hombres y } 293 \\
\text { mujeres (edad media de } \\
20,1 \text { años) }\end{array}$ & $\begin{array}{l}\text { Consumo de } 5 \text { ó más bebidas ( } 4 \text { para mujeres) en una única } \\
\text { ocasión en los últimos tres meses. }\end{array}$ \\
\hline
\end{tabular}

\begin{tabular}{|c|c|c|c|c|}
\hline $\begin{array}{l}\text { Townshend y Duka, } \\
2002^{67}\end{array}$ & $\begin{array}{l}\text { Inglat- } \\
\text { erra }\end{array}$ & $\begin{array}{l}\text { Comprobar si el AUQ es más preciso que el } \\
\text { diario de consumo para detectar la pres- } \\
\text { encia de CIA. }\end{array}$ & $\begin{array}{l}14 \text { hombres y } 42 \text { mujeres } \\
\text { estudiantes universitarios } \\
\text { (edad media de } 20,54 \\
\text { años). }\end{array}$ & $\begin{array}{l}\text { Puntuación } \geq \text { a } 24 \text { en los ítems } 10 \text { (rapidez de consumo - } \\
\text { media de bebidas/hora), } 11 \text { (cantidad de borracheras en los } \\
\text { últimos } 6 \text { meses) y } 12 \text { (\% de borracheras en dias de con- } \\
\text { sumo) del AUQ ( } 4 \text { × (item 10) + item } 11 \text { + } 0.2 \text { x (item 12). }\end{array}$ \\
\hline
\end{tabular}

$\begin{array}{ll}\text { Jennison, 2004 } & \text { Determinar si el patrón CIA en estudiantes } \\ & \text { es un factor de riesgo para el futuro abuso/ } \\ \text { dependencia al alcohol. }\end{array}$

1.003 hombres y 969 mujeres universitarios (edad media de 21 años).
Consumo de 5 ó más bebidas por ocasión para hombres (4 ó más para mujeres) al menos una vez en el último mes.

\begin{tabular}{|c|c|c|c|c|}
\hline McCarty et al., 2004 ${ }^{68}$ & EEUU & $\begin{array}{l}\text { Determinar la asociación entre el CIA en la } \\
\text { adolescencia tardia y el consumo en la vida } \\
\text { adulta temprana. }\end{array}$ & $\begin{array}{l}1.888 \text { hombres y } 1.902 \\
\text { mujeres evaluados entre } \\
\text { los } 17 \text { - } 20 \text { años entre los } \\
\text { 30-31 años. }\end{array}$ & $\begin{array}{l}\text { Consumo de } 6 \text { ó más bebidas en una ocasión al menos una } \\
\text { vez en los últimos } 30 \text { días. }\end{array}$ \\
\hline Daeppen et al., $2005^{69}$ & Suiza & $\begin{array}{l}\text { Describir el consumo de alcohol, CIA y las } \\
\text { consecuencias asociadas en hombres de } \\
\qquad 19 \text { años. }\end{array}$ & $\begin{array}{l}1.004 \text { hombres realizando } \\
\text { el servicio militar (edad } \\
\text { media de } 19 \text { años). }\end{array}$ & $\begin{array}{l}\text { Consumo de } 5 \text { ó más bebidas por ocasión al menos una vez } \\
\text { en los últimos } 12 \text { meses. }\end{array}$ \\
\hline $\begin{array}{l}\text { Guilamo-Ramos et al., } \\
\qquad 2005^{70}\end{array}$ & EEUU & $\begin{array}{l}\text { Estimar la prevalencia de CIA entre estudi- } \\
\text { antes de enseñanza secundaria. }\end{array}$ & $\begin{array}{l}5.300 \text { estudiantes entre } 12 \\
\text { y } 14 \text { años. }\end{array}$ & $\begin{array}{l}\text { Consumo de } 5 \text { ó más bebidas por ocasión al menos una vez } \\
\text { en los últimos } 12 \text { meses. }\end{array}$ \\
\hline Nelson, et al., $2005^{71}$ & EEUU & $\begin{array}{l}\text { Determinar en qué medida las tasas de CIA } \\
\text { en universitarios están relacionadas con las } \\
\text { tasas de CIA en la población general y con } \\
\text { las políticas estatales de control de alcohol. }\end{array}$ & $\begin{array}{c}\text { CAS: } 22.453 \text { estudi- } \\
\text { antes, entre } 1824 \text { años } \\
\text { vs. BRFSS: } 352.266 \text {, de } 18 \\
\text { años o más, de los cuales } \\
31.042 \text { eran jóvenes de } 18 \\
\text { a } 24 \text { años. }\end{array}$ & $\begin{array}{l}\text { CAS: Consumo de } 5 \text { ó más bebidas por ocasión para } \\
\text { hombres ( } 4 \text { ó más para mujeres) al menos una vez en los } \\
\text { últimos quince dias. BRFSS: Consumo de } 5 \text { ó más bebidas } \\
\text { alcohólicas por ocasión al menos una vez en el último mes. }\end{array}$ \\
\hline
\end{tabular}


Tabla 1. Estudios de consumo intensivo de alcohol en adolescentes y/o universitarios (continuación).

\begin{tabular}{lllll}
\hline ESTUDIO & PAIS & OBJETIVO & SUJETOS & CRITERIO DE CIA \\
\hline Bendtsen et al., 20065 & Suecia & $\begin{array}{l}\text { Viabilidad del empleo un cuestionario via } \\
\text { correo electrónico en universitarios. }\end{array}$ & $\begin{array}{l}843 \text { hombres y 742 } \\
\text { mujeres estudiantes. }\end{array}$ & $\begin{array}{l}\text { Consumo de } 5 \text { bebidas (4 para mujeres) en una única } \\
\text { ocasión una vez al mes. }\end{array}$ \\
\hline Cranford et al., 200653 & EEUU & $\begin{array}{l}\text { Comparar la definición operativa de } \\
\text { Wechsler et al. (1994) con la de la NIAAA } \\
\text { (2004). }\end{array}$ & $\begin{array}{l}\text { 2.276 hombres y 2.304 } \\
\text { mujeres sujetos (edad } \\
\text { media de 19,9 años). }\end{array}$ & $\begin{array}{l}\text { Consumo de } 5 \text { ó más bebidas por ocasión (4 para mujeres) } \\
\text { (a) al menos una vez en los últimos quince dias; } \text { (b) en un } \\
\text { periodo de dos horas en los últimos 12 meses. }\end{array}$
\end{tabular}

\begin{tabular}{|c|c|c|c|c|}
\hline D'Alessio et al., $2006^{72}$ & Italia & $\begin{array}{l}\text { Valorar la prevalencia de CIA en estudi- } \\
\text { antes universitarios }\end{array}$ & $\begin{array}{l}358 \text { hombres y } 342 \\
\text { mujeres (edad media de } \\
22,59 \text { años). }\end{array}$ & $\begin{array}{l}\text { Consumo de } 5 \text { ó más bebidas ( } 4 \text { para mujeres), en una } \\
\text { única ocasión, una o dos veces en la última semana. }\end{array}$ \\
\hline Griffiths et al., $2006^{73}$ & China & $\begin{array}{l}\text { Estudiar el consumo de alcohol en } \\
\text { jóvenes universitarios. }\end{array}$ & $\begin{array}{l}1.211 \text { hombres y } 1.402 \\
\text { mujeres (edad media de } \\
18,9 \text { años). }\end{array}$ & $\begin{array}{l}\text { Consumo de } 5 \text { ó más bebidas en una única ocasión en el } \\
\text { último mes. }\end{array}$ \\
\hline
\end{tabular}

$\begin{array}{llll}\text { Isralowitz et al., 2006 } & \text { Israel } & \begin{array}{l}\text { Medir la prevalencia de CIA en estudi- } \\ \text { antes de secundaria }\end{array} & \begin{array}{l}394 \text { hombres y } 523 \\ \text { mujeres (edad media de } \\ 16 \text { años) }\end{array}\end{array} \begin{aligned} & \text { Consumo de } 5 \text { ó más bebidas, en una única ocasión, al } \\ & \text { menos una vez en el último mes. }\end{aligned}$

\begin{tabular}{|c|c|c|c|c|}
\hline White et al., 2006 ${ }^{17}$ & EEUU & $\begin{array}{l}\text { Examinar la presencia de CIA en estudi- } \\
\text { antes universitarios. }\end{array}$ & $\begin{array}{l}5.004 \text { hombres y } 5.420 \\
\text { mujeres (edad media de } \\
18,14 \text { años). }\end{array}$ & $\begin{array}{l}\text { Consumo de } 5 \text { ó más bebidas por ocasión para hombres ( } 4 \\
\text { para mujeres), al menos una vez en los últimos } 15 \text { dias. }\end{array}$ \\
\hline Xing et al., $2006^{75}$ & China & $\begin{array}{l}\text { Describir la frecuencia y los patrones } \\
\text { de consumo de alcohol y observar la } \\
\text { asociación entre CIA y la salud de ado- } \\
\text { lescentes. }\end{array}$ & $\begin{array}{l}24.318 \text { hombres y } 29.722 \\
\text { mujeres (edad media de } \\
13,8 \text { años). }\end{array}$ & $\begin{array}{l}\text { Consumo de } 5 \text { ó más bebidas por ocasión, al menos una } \\
\text { vez en el último mes. }\end{array}$ \\
\hline
\end{tabular}

\begin{tabular}{|c|c|c|c|c|}
\hline Gill, et al., 200776 & Escocia & $\begin{array}{l}\text { Estudio de los aspectos cualitativos para } \\
\text { establecer un patrón de CIA. }\end{array}$ & $\begin{array}{l}95 \text { mujeres estudiantes } \\
\text { universitarias (edad media } \\
\text { de } 20,1 \text { años). }\end{array}$ & $\begin{array}{l}\text { Consumo de } 7 \text { ó más bebidas en una ocasión, al menos una } \\
\text { vez en la última semana. }\end{array}$ \\
\hline
\end{tabular}

\begin{tabular}{|c|c|c|c|c|}
\hline Keller, et al., $2007^{77}$ & Alemania & $\begin{array}{l}\text { Relacionar las normas sociales } \\
\text { percibidas/actitudes hacía el consumo } \\
\text { de alcohol y patrones de CIA. }\end{array}$ & $\begin{array}{l}96 \text { hombres y } 156 \\
\text { mujeres estudiantes uni- } \\
\text { versitarios (edad media de } \\
20,6 \text { años). }\end{array}$ & $\begin{array}{l}\text { Consumo de } 5 \text { ó más bebidas por ocasión ( } 4 \text { para mujeres), } \\
\text { al menos una vez en los últimos } 15 \text { días. }\end{array}$ \\
\hline $\begin{array}{l}\text { Caamaño-Isorna, et al., } \\
2008^{78}\end{array}$ & España & $\begin{array}{l}\text { Estimar la prevalencia de un patrón de } \\
\text { CIA en estudiantes universitarios. }\end{array}$ & $\begin{array}{l}371 \text { hombres y } 992 \\
\text { mujeres entre } 18 \text { y } 19 \\
\text { años. }\end{array}$ & $\begin{array}{l}\text { Consumo de } 6 \text { ó más bebidas en una única ocasión una vez } \\
\text { a la semana. }\end{array}$ \\
\hline
\end{tabular}

\begin{tabular}{|c|c|c|c|c|}
\hline Page, et al., $2008^{79}$ & $\begin{array}{l}\text { Hungria, } \\
\text { Eslovaquia, } \\
\text { Chequia y } \\
\text { Rumania }\end{array}$ & $\begin{array}{l}\text { Valorar la influencia de consumo de los } \\
\text { iguales en el propio consumo. }\end{array}$ & 1.886 estudiantes. & $\begin{array}{l}\text { Consumo de } 5 \text { ó más bebidas, en dos horas, al menos una } \\
\text { vez en los últimos } 30 \text { dias. }\end{array}$ \\
\hline Kypri et al., $2009^{80}$ & $\begin{array}{l}\text { Nueva } \\
\text { Zelanda }\end{array}$ & $\begin{array}{l}\text { Estimar la prevalencia de CIA y factores } \\
\text { de riesgo asociados en estudiantes } \\
\text { universitarios. }\end{array}$ & $\begin{array}{l}2.548 \text { estudiantes (edad } \\
\text { media de 20,2 años). }\end{array}$ & $\begin{array}{l}\text { Consumo de } 6 \text { ó más bebidas alcohólicas por ocasión/4 ó } \\
\text { más para mujeres durante el último año. }\end{array}$ \\
\hline
\end{tabular}

Nota: CIA (Consumo Intensivo de Alcohol); NYRBS (National Youth Risk Behavior Survey); CAS (College Alcohol Survey); BRFSS (Behavioral Risk Factor Surveillance System); NLSY (National Surveys of Youth); MTF (Monitoring The Future). 
estadounidense ${ }^{20}$, diversos autores han mostrado el alarmante incremento del consumo de alcohol entre jóvenes universitarios, empezando a plantearse la existencia de un patrón de consumo de riesgo en esta población ${ }^{21-24}$.

En la década de los setenta, el estudio epidemiológico Monitoring the Future Study $y^{23}$ introduce el término binge drinking para referirse a un patrón de consumo prevalente en la población entre los 18 y los 24 años (sobre todo antes de los 21 años), caracterizado por la ingesta de grandes cantidades de alcohol en un corto período de tiempo. Los autores lo definieron como el consumo de 5 ó más bebidas alcohólicas en una única ocasión, en las dos últimas semanas. Este punto de corte se estableció basándose en estudios previos que consideraban este umbral como significativamente relacionado con problemas asociados al consumo de alcohol.

En los años noventa, a partir de una investigación del Harvard School of Public Health College Alcohol Study ${ }^{20,25}$, se identificó la importancia del sexo de los sujetos en los efectos del consumo de alcohol. El grupo de Wechsler, utilizando un cuestionario de hábitos de consumo de alcohol, encontró que los hombres muestran de forma significativa problemas relacionados con el consumo de alcohol a partir de 5 bebidas por ocasión y las mujeres a partir de 4 bebidas. Así se operativizó el concepto de ClA como el consumo de 5 ó más en varones y 4 ó más en mujeres en una única ocasión, al menos una vez en las últimas dos semanas ${ }^{25}$.

Aunque la propuesta de Wechsler et al. ${ }^{25}$ está bastante extendida para definir el patrón de CIA, existe una gran controversia en torno a ella, llegando a sugerirse que esta terminología puede falsear el alcance del problema del consumo intensivo de alcohol entre estudiantes universitarios ${ }^{26}$. Las críticas se centran en aspectos tales como: a) la propia conceptualización del término anglosajón binge drinking; b) el establecimiento de cantidades de alcohol demasiado pequeñas para calificarlas como consumo problemático de alcohol; c) la falta de definición de una bebida estándar; y d) la no especificación del período de tiempo que se considera "una única ocasión".

En relación con la controversia existente acerca del término anglosajón binge drinking, diversos autores señalan que tradicionalmente este concepto ha hecho referencia a un patrón de consumo intensivo de alcohol en un largo período de tiempo (de al menos dos días seguidos), que interfiere de forma significativa en la vida de la persona, relacionándolo más con definiciones clínicas de abuso y/o dependencia ${ }^{27-29}$. Por este motivo sugieren términos alternativos como heavy drinking ${ }^{30-34}$, heavy episodic drinking ${ }^{35-40}$, heavy sessional drinking ${ }^{41}$, risky single-occasion drinking ${ }^{42}$, dangerous drinking ${ }^{26}, 0$ high-risk drinking ${ }^{43}$ para evitar la confusión en relación al término. En España, en la Primera Conferencia de Prevención y Promoción de la Salud en la Práctica Clínica realizada en el 2007 se ha propuesto el siguiente término: episodios de consumo intensivo de alcohol ${ }^{14}$.
Sin embargo, el grupo de Wechsler defiende este término argumentando que "binge"o atracón se utiliza comúnmente para referirse a acciones como comer o comprar compulsivamente (binge eating; binge buying respectivamente), donde la duración no es un prerrequisito ${ }^{44}$. De esta manera su aplicación referida al consumo intensivo de alcohol es consistente con este significado comúnmente aceptado. Así, se puede considerar que el término anglosajón binge drinking tiene dos acepciones claramente diferenciadas: una relacionada con definiciones clínicas de alcoholismo (abuso/dependencia al alcohol) e indicadora de problemas relacionados con el consumo de alcohol; y otra referida al consumo excesivo de alcohol ocasional en la población en general ${ }^{29}$.

En cuanto al establecimiento de la cantidad de alcohol, se ha cuestionado tanto el umbral establecido, como el hecho de que la definición implícitamente asume iguales riesgos ante cualquier consumo que supere el umbral establecido. En este sentido, White et al. ${ }^{17}$, examinando los patrones de consumo de jóvenes universitarios, encontraron que un alto porcentaje de estudiantes consumian por encima de los umbrales propuestos por Wechsler et al. ${ }^{20,25}$, llegando incluso a doblarlos. En concreto, se comprobó que 1 de cada 5 varones universitarios habían consumido 10 ó más bebidas alcohólicas y 1 de cada 10 mujeres 8 ó más bebidas, al menos una vez en las dos últimas semanas.

Ante estos datos, Wechsler y Nelson argumentan que aumentar el umbral de riesgo a 10 para hombres y 8 para mujeres sería válido para determinar de forma precisa quién podría cumplir los criterios de DSM-IV de abuso y/o dependencia al alcohol pero podría no resultar adecuado para establecer un punto de corte de riesgo de la población general ${ }^{45}$.

Sin embargo, un estudio reciente ${ }^{46}$, en el que se comparaba a tres grupos de estudiantes, no CIA (menos de 5 ó 4 bebidas); CIA típico (consumo de 5 ó 4); y CIA duro (consumo de 7 ó 6), mostró, en la línea de la investigación Ilevada a cabo por White et al. ${ }^{17}$, que el punto de corte $5 / 4$ no es suficiente para determinar un patrón de riesgo en la población universitaria. Esto se debe a que los sujetos del grupo CIA duro son los que experimentan significativamente más consecuencias negativas relacionadas con el consumo de alcohol en comparación con los otros dos grupos. Ante este resultado, los autores se plantean cuál sería el porcentaje de consumidores intensivos de alcohol duros en los estudios del grupo de Wechsler ${ }^{20,24,25}$, resurgiendo el debate acerca de si 5 bebidas - 4 para mujeres- es un punto de corte suficiente para establecer un patrón de consumo de riesgo en la población universitaria.

Otra de las críticas a la vigente conceptualización del CIA es la falta de definición de una UBE. La UBE varía considerablemente de unos países a otros, por lo que es necesario considerar las diferencias de etanol puro en cada caso $^{47}$ (Tabla 2). 
Tabla 2. Gramos de alcohol por unidad de bebida estándar (adaptado de ICAP, 2003) ${ }^{46}$.

\begin{tabular}{lc}
\hline País & $\begin{array}{c}\text { Gramos de } \\
\text { alcohol }\end{array}$ \\
\hline Reino Unido & 8 \\
Países Bajos & 9,9 \\
$\begin{array}{l}\text { Australia, Austria, Irlanda, Nueva Zelanda, } \\
\text { Polonia y España }\end{array}$ & 10 \\
Finlandia & 11 \\
Dinamarca, Francia, Italia y Sudáfrica & 12 \\
Canadá & 13,6 \\
Portugal, EEUU & 14 \\
Japón & 19,75 \\
\hline
\end{tabular}

Estas diferencias obligan a adaptar el criterio de 5/4 al país en el que se realice el estudio. Así, por ejemplo, mientras que en Portugal se podría emplear el mismo punto de corte que en EEUU, la UBE utilizada en Reino Unido obliga a definir el CIA como el consumo de 8 ó más bebidas para hombres y 6 ó más para mujeres en una única ocasión en la última semana ${ }^{48,49}$.

Por último, el concepto "única ocasión" presenta también limitaciones. Diversos autores plantean la necesidad de tener en cuenta los niveles de concentración de alcohol en sangre (CAS) para determinar de forma precisa cuál es el umbral adecuado para establecer un patrón de CIA. Esto responde a que la ingesta de una bebida alcohólica por hora durante cinco horas seguidas no produce los mismos efectos en un adulto de peso corporal medio, que el consumo de la misma cantidad de alcohol ( 5 bebidas en total) en un período de, por ejemplo, dos horas.

En este sentido, el National Institute on Alcohol Abuse and Alcoholism (NIAAA) ${ }^{50}$ redefinió el término CIA tomando en consideración el nivel de concentración de alcohol en sangre. Así, CIA implica un patrón de consumo de alcohol que eleva el nivel al menos al 0,08 g/L. En adultos, esto equivale a 5 ó más bebidas para hombres y 4 ó más bebidas para mujeres consumidas en aproximadamente dos horas. De esta manera el NIAAA tiene en cuenta la variable duración (dos horas) en la definición de CIA.

Diversos estudios han empleado diferentes variantes de la fórmula desarrollada en los años 30 por Widmark para estimar la concentración de alcohol en sangre de forma retrospectiva. Esta ecuación establece que la concentración máxima de alcohol en sangre es $A /(p \times r)$, siendo $A=$ Cantidad de alcohol consumida (en gramos); $P=$ masa corporal y $\mathrm{R}=$ proporción agua/grasa $(0,7$ para hombres y 0,6 para mujeres).
El empleo de esta fórmula ha mostrado una adecuada fiabilidad ${ }^{51}$. Para ello, se ha propuesto una alternativa a las cuestiones prototípicas de cantidad y frecuencia: el diario de consumo. Este instrumento recoge el número de bebidas consumidas en los últimos días (normalmente siete días). De esta manera es posible conocer la duración (en horas) de cada episodio de consumo y la ingesta de una semana típica. Si a esto se añaden los datos de sexo y peso corporal, se obtienen las variables necesarias para estimar la concentración de alcohol en sangre ${ }^{46,52}$.

Recientemente, el estudio de Cranford et al. ${ }^{53}$ señala que la definición del grupo de Wechsler ${ }^{20,25}$ y la propuesta por el NIAAA ${ }^{50}$ presentan una alta correlación y un patrón similar de asociación con las variables sexo, raza y edad de inicio de consumo. Sin embargo, estos autores consideran que es mejor tener en cuenta la cantidad y la duración como defiende la NIAAA, ya que considerar sólo la variable cantidad está infraestimando la prevalencia de CIA, no siendo lo suficientemente sensible para la discriminación entre patrones de consumo problemáticos y no problemáticos.

\section{Frecuencia de consumo}

Debido a la variabilidad de los sujetos en la frecuencia de consumo, se hace necesario considerar este aspecto para la definición del CIA. El estudio de Wechsler et al. ${ }^{20}$, definió cuatro grupos de sujetos en función de la frecuencia de consumo: (1) Abstinentes: aquellos que no han consumido alcohol en el último año; (2) No CIA: los que consumieron alcohol en el último año, pero no intensamente en las dos últimas semanas; (3) CIA ocasional: aquellos que han consumido alcohol de forman intensiva 1 ó 2 veces en las dos últimas semanas; (4) CIA frecuente: los que han consumido alcohol de forma intensiva 3 ó más veces en las dos últimas semanas. Wechsler et al. ${ }^{20}$ encontraron que, de los 17.592 estudiantes universitarios encuestados, el $44 \%$ se podrían clasificar como consumidores intensivos de alcohol y de estos, el 19\% como consumidores intensivos de alcohol frecuentes.

Sin embargo, un estudio más reciente ${ }^{54}$ sugiere que en torno al $6 \%$ de los estudiantes universitarios cumplen los criterios de abuso y/o dependencia al alcohol del DSM-IV, y esto es particularmente alto en los consumidores intensivos frecuentes (grupo en el cual sobre un 20\% podrían cumplir los criterios). Así, probablemente, un alto porcentaje de los sujetos clasificados como consumidores intensivos frecuentes en el estudio de Wechsler et al. ${ }^{20}$ podrían cumplir Ios criterios de DSM-IV de abuso y/o dependencia del alcohol. De este modo, persiste la controversia acerca de cuáles son los límites para establecer un consumo de riesgo referido a la población general, sin llegar a criterios de abuso/dependencia al alcohol.

Existe igualmente controversia sobre la utilización de dos semanas como intervalo de tiempo para determinar la presencia de CIA. Considerar sólo lo que ocurre en las dos últimas semanas puede infraestimar la prevalencia de $\mathrm{CIA}$, debido a la variabilidad que muestran los estudiantes en su 
frecuencia de consumo a lo largo del curso académico. En este sentido, Vik et al. ${ }^{55}$ encontraron que la prevalencia en hombres pasaba del $50 \%$ al $77,5 \%$ y del $47,2 \%$ al $79,2 \%$ en mujeres cuando se cambiaba el período de medida de 2 semanas a tres meses. Atendiendo a las consecuencias negativas asociadas al consumo de alcohol, el grupo de Vik ${ }^{55}$ no encuentra diferencias significativas entre el grupo de dos semanas y el de tres meses en el número de problemas relacionados con el alcohol en el último año. Esto sugiere que emplear un intervalo de tres meses puede proporcionarnos datos más fiables sobre la prevalencia de CIA.

En la misma línea, un estudio llevado a cabo por Labrie et al. ${ }^{31}$ en el que se analizaba si existía variabilidad en intervalos de dos semanas durante el último mes, encontraron que un tercio de los estudiantes universitarios clasificados como No CIA en las dos últimas semanas del mes, se clasificarian como ClA en las dos primeras semanas. Además, este grupo, al que denominaron grupo inconsistente, presentaba un alto rango de consecuencias negativas asociadas al consumo de alcohol, similares a los que mostraban los clasificados como CIA.

Por lo tanto, del mismo modo que no existe un criterio unánime para operativizar el concepto $\mathrm{CIA}$ en términos de cantidad, tampoco existe uno claro para el establecimiento de la frecuencia como criterio de riesgo, mostrándose una gran variabilidad entre los diferentes estudios al adoptar un criterio de frecuencia de consumo.

\section{Consecuencias negativas asociadas al consumo de alcohol}

En los últimos veinte años diversos estudios han observado que el CIA se relaciona con un incremento de las consecuencias negativas asociadas al consumo de alcohol (como accidentes de tráfico, suicidios, violencia y delitos, enfermedades de transmisión sexual o bajo rendimiento académico) ${ }^{56,57,58}$. Incluso algunas investigaciones han comprobado que esta forma de consumo se asocia con consecuencias neurocognitivas (como peor rendimiento en tareas neuropsicológicas de memoria o toma de decisiones o deficiencia en la diferenciación electrofisiológica entre información relevante e irrelevante) ${ }^{59,60,61}$. Sin embargo, aunque son numerosos los estudios que, tanto al definir el concepto de $\mathrm{CIA}$ en términos del número de bebidas como en función de la frecuencia de su consumo, establecen un punto de corte basándose, entre otros aspectos, en las consecuencias negativas asociadas al consumo de alcohol, la mayoría de ellos no lo incluye explícitamente en la definición operativa de $\mathrm{CI}^{19}$.

Uno de los escasos estudios que sí ha considerado esta variable en la definición es el desarrollado por Baer et al. ${ }^{43}$. Estos autores definen CIA como el consumo de 5-6 bebidas alcohólicas al menos una vez al mes y la presencia de al menos tres consecuencias negativas asociadas al consumo de alcohol entre tres y cinco veces en los últimos tres años.

Probablemente el motivo por que el que muy pocos estudios hayan considerado la importancia de esta variable a la hora de operativizar el concepto de CIA radique en que las variables cantidad y frecuencia de consumo nos pueden aportar esta información de manera implícita ${ }^{19}$. Así, por ejemplo, el punto de corte establecido por el grupo de Wechsler se basó, entre otros aspectos, en las consecuencias negativas asociadas al consumo de alcohol ${ }^{25}$. Estos autores, administrando un cuestionario de hábitos de consumo de alcohol, comprobaron que los hombres mostraban de forma significativa problemas relacionados con el consumo de alcohol a partir de cinco bebidas por ocasión, y las mujeres a partir de cuatro bebidas. Del mismo modo, la frecuencia de consumo también nos aporta información al respecto ya que, aunque con algún estudio discrepante, aquellos jóvenes que presentan este patrón de consumo con mayor frecuencia son los que generalmente muestran más consecuencias negativas asociadas al consumo de alcohol ${ }^{19}$.

Un aspecto muy importante a la hora de establecer el criterio operativo del CIA es diferenciarlo del criterio de abuso/dependencia de alcohol. Existe un consenso general en tratarlos como categorías excluyentes, aunque relacionadas. El CIA se considera un patrón de consumo de la población general, en el que los sujetos no cumplen los criterios establecidos en los manuales diagnósticos de referencia para el trastorno de abuso/dependencia del alcohol. Sin embargo, ambos están relacionados. Diversos estudios han observado que el CIA durante la adolescencia se asocia con desarrollo posterior de abuso/dependencia del alcohol en la edad adulta ${ }^{11,12,13}$, siendo los consumidores intensivos que presentan este patrón de forma más frecuente (3 ó más veces en las dos últimas semanas) los que tienen más probabilidad de presentar abuso/dependencia ${ }^{20}$. Aunque en el diagnóstico de este trastorno se tengan en cuenta muchas de las consecuencias negativas, también presentes en la población adolescente general que consume alcohol de forma intensiva, no es adecuado establecer una equivalencia entre ambos. La prevalencia y gravedad de tales consecuencias es menor entre los sujetos con consumo intensivo de alcohol, debido en buena medida a las características de cantidad y frecuencia del patrón de consumo.

En suma, el estudio del patrón de CIA requiere tener en cuenta las variables cantidad, frecuencia, además de tener presentes las consecuencias negativas asociadas. La combinación de estas variables, así como la adaptación al país dónde se realice el estudio es lo que dificulta el establecimiento de una definición operativa unánime del término CIA. Esta falta de consenso ha llevado a algunos autores a optar por emplear una aproximación cualitativa a la hora de establecer un patrón de $\mathrm{CI} A$, basándose en aspectos como el número de veces que se ha emborrachado o los efectos del consumo de alcohol en el comportamiento de cada persona ${ }^{62-65}$.

En España, desde el ámbito preventivo, se ha propuesto una posible definición operativa del consumo intensivo de alcohol que contempla la cantidad ingerida pero no la frecuencia de consumo ${ }^{14}$. Una definición complementaria que podría resultar más adecuada en el ámbito clínico podría ser la siguiente: consumo de 6 ó más bebidas alcohólicas para hombres (60 g) -5 ó más para mujeres (50 g)- en una 
única ocasión (en un período de dos horas) al menos una vez en los últimos 30 días. Además de aproximarse al enfoque adoptado por el $\operatorname{NIAAA}^{50}$ y el grupo de Wechsler ${ }^{20,25}$, esta definición recoge todas las variables que se han mostrado más relevantes en el intento de operativizar el término CIA (cantidad y frecuencia), sin olvidar la necesidad de adaptarlo al país donde se pretende realizar la investigación.

\section{AGRADECIMIENTOS}

Esta investigación ha sido financiada por la Conselleria de Innovación e Industria de la Xunta de Galicia (INCITE08PXIB211015PR), por el Plan Nacional sobre Drogas del Ministerio de Salud y Consumo (2005/PN014) y por el Ministerio de Ciencia e Innovación (EDU2008-03400). Los autores M. Parada, N. Mota y A.Crego han contado con una beca predoctoral de la Diputación de A Coruña, la Consellería de Innovación e Industria de la Xunta de Galicia, y del Ministerio de Educación, respectivamente.

\section{REFERENCIAS}

1 Delegación del gobierno para el Plan Nacional sobre Drogas. Informe sobre alcohol [Citado 10 enero de 2007]. Disponible en: http://www.pnsd.msc.es/Categoria2/publica/pdf/InformeAlcohol. pdf

2 Organización Mundial de la Salud. Problemas de salud pública causados por el uso nocivo del alcohol [Citado 27 octubre de 2005]. Disponible en: http//www.who.int/substance_abuse/ who_resolution_58_26_public_health_problems_alcohol_ spanish-pdf

3 Sánchez-Pardo L. Situación actual y evolución de los consumos de drogas ilícitas en España. Trast Adict 2001;3 :85-94.

4 Sánchez-Pardo L. Consumo alcohólico e la población española. Adicciones 2002; 14: 79-97.

5 Delegación del gobierno para el Plan Nacional sobre Drogas. Informe 2004 del Observatorio Español sobre Drogas. Situación y tendencias de los problemas de drogas en España. [Citado el 27 de Marzo de 2005].Disponible en: http://www.pnsd.msc.es/ Categoria2/publica/pdf/oed-2004.pdf

6 Delegación del Gobierno para el Plan Nacional sobre Drogas. Informe de la encuesta domiciliaria sobre alcohol y drogas de España [Citado 4 abril de 2009]. Disponible en: http://www.pnsd. msc.es/Categoria2/observa/pdf/InformeEdades2007-2008.pdf

7 Bloomfield K, Stockwell T, Gmel G, Rehn N. International comparisons of alcohol consumption. Alcohol Res Health 2003; 27: 95-109.

8 Kuntsche $E_{1}$ Rehm J, Gmel G. Characteristics of binge drinkers in Europe. Soc Sci Med 2004; 59: 113-127.
9 Perkins HW. Surveying the damage: a review of research on consequences of alcohol misuse in college populations. J Stud Alcohol 2002; 14: 91-100.

10 Room R, Babor T, Rehm J. Alcohol and public health. Lancet 2005; 365: 519-30.

11 Grant BF, Dawson DA, Stinson FS, Chou SP, Dufour MC, Pickering RP. The 12-month prevalence and trends in DSM-IV alcohol abuse and dependence: United States, 1991-1992 and 20012002. Drug Alcohol Depend 2004; 74: 223-34.

12 Grant JD, Scherrer JF, Lynskey MT, Lyons MJ, Eisen SA, Tsuang $M T$, et al. Adolescent alcohol use is a risk factor for adult alcohol and drug dependence: evidence from a twin design. Psychol Med 2006; 36: 109-18.

13 Jennison KM. The short-term effects and unintended long-term consequences of binge drinking in college: a 10-year follow-up study. Am J Drug Alcohol Abuse 2004; 30: 659-84.

14 Ministerio de Sanidad y Consumo. Prevención de los problemas derivados del alcohol: Primera Conferencia de Prevención y Promoción de la Salud en la Práctica Clínica en España [Citado 5 diciembre de 2008]. Disponible en: http://www. msc.es/profesionales/saludPublica/prevPromocion/docs/ prevencionProblemasAlcohol.pdf

15 Hibell B, Anderson B, Bjarnason T, Ahlstrom S, Balakireva O, Kokkevi A, Morgan M. The ESPAD report 2003. Alcohol and other drugs use among students in 35 european countries. The Swedish council for information and other drugs (CAN) and the Pompidou Group at the concil of Europe. Sweden: Stockholm; 2004.

16 Newes-Adeyi G, Chen CM, Williams GD, Fader VB. Trends in underage drinking in the United States, 1991-2003, Surveillance Report No. 70. Bethesda, MD: Division of Epidemilogy and Prevention Research, National Institute on Alcohol Abuse and Alcoholism, 2005.

17 White A, Kraus CL, Swartzwelder, H. Many college freshmen drink at levels far bayond the binge threshold. Alcohol Clin Exp Res 2006; 30: 1006-10.

18 Organización Mundial de la Salud. Global Status Report on Alcohol 2004. Geneva, Switzerland: Department of Mental Health and Substance Abuse, World Health Organization, 2004.

19 Ham LS, Hope DA. College students and problematic drinking: a review of the literature. Clin Psychol Rev 2003; 23: 719-59.

20 Wechsler H, Davenport A, Dowdall G, Moeykens B, Castillo $S$. Health and behavioral consequences of binge drinking in college. A national survey of students at 140 campuses. JAMA 1994; 272: 1672-77.

21 Anderson P. Binge drinking and Europe. London: Institute of Alcohol Studies. 2007.

22 National Institute on Alcohol Abuse and Alcoholism. A call to action: changing the culture of drinking at U.S. colleges 
[Citado 10 de diciembre de 2002]. Disponible en: http// www.collegedrinking prevention.gov/NIAAACollegeMaterials/ TaskForce_TOC.aspx.

23 O'Malley PM, Bachman JG, Johnston LD. Period, age, and cohort effects on substance use among American youth 1976-1982. Michigan: Institute for Social Research. 1984.

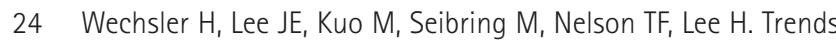
in college binge drinking during a period of increased prevention efforts. Findings from 4 Harvard School of Public Health College Alcohol Study surveys: 1993-2001. J Am Coll Health 2002; 50 : 203-17.

25 Wechsler H, Dowdall GW, Davenport A, Rimm EB. A genderspecific measure of binge drinking among college students. Am J Public Health 1995: 85: 982-5.

26 Lederman LC, Stewart LP, Goodhart FW, Laitman L. A case against "binge" as the term of choice: convincing college students to personalize messages about dangerous drinking. $J$ Health Commun 2003; 8: 79-91.

27 Epstein EE, Kahler CW, McCrady BS, Lewis KD, Lewis S. An empirical classification of drinking patterns among alcoholics: binge, episodic, sporadic, and steady. Addict Behav 1995; 20: 23-41.

28 Gill JS. Reported levels of alcohol consumption and binge drinking within the UK undergraduate student population over the last 25 years. Alcohol Alcoholism 2002; 37: 109-120.

29 Lange JE, Clapp JD, Turrisi R, Reavy R, Jaccard J, Johnson MB, et al. College binge drinking: what is it? Who does it? Alcohol Clin Exp Res 2002; 26: 723-30.

30 Christiansen M, Vik PW, Jarchow A. College student heavy drinking in social contexts versus alone. Addict Behav 2002; 27: 393-404.

31 LaBrie JW, Pedersen ER, Tawalbeh S. Classifying risky-drinking college students: another look at the two-week drinker-type categorization. J Stud Alcohol 2007; 68: 86-90.

32 Sher KJ, Rutledge PC. Heavy drinking across the transition to college: predicting first-semester heavy drinking from precollege variables. Addict Behav 2007; 32: 819-835.

33 Turrisi R, Mallett KA, Mastroleo NR, Larimer ME. Heavy drinking in college students: who is at risk and what is being done about it? J Gen Psychol 2006; 133: 401-420.

34 Vik PW, Carrello P, Tate SR, Field C. Progression of consequences among heavy-drinking college students. Psychol Addict Behav 2000; 14: 91-101.

35 Bendtsen $\mathrm{P}$, Johansson $K_{1}$ Akerlind I. Feasibility of an emailbased electronic screening and brief intervention (e-SBI) to college students in Sweden. Addict Behav 2006; 31: 777-87.
36 Collins SE, Carey KB. The theory of planned behavior as a model of heavy episodic drinking among college students. Psychol Addict Behav 2007; 21: 498-507.

37 Dawson DA, Grant BF, Stinson FS, Chou PS. Another look at heavy episodic drinking and alcohol use disorders among college and noncollege youth. J Stud Alcohol 2004; 65: 477488.

38 Makela P, Fonager K, Hibell B, Nordlund S, Sabroe S, Simpura J. Episodic heavy drinking in four Nordic countries: a comparative survey. Addiction 2001; 96: 1575-88.

39 Oesterle S, Hill KG, Hawkins JD, Guo J, Catalano RF, Abbott RD. Adolescent heavy episodic drinking trajectories and health in young adulthood. J Stud Alcohol 2004; 65: 204-212.

40 Wilsnack RW, Vogeltanz ND, Wilsnack SC, Harris TR, Ahlstrom $\mathrm{S}$, Bondy $\mathrm{S}$, et al. Gender differences in alcohol consumption and adverse drinking consequences: cross-cultural patterns. Addiction 2000; 95: 251-65.

41 Measham F. The "big bang" approach to sessional drinking: changing patterns of alcohol consumption amongst young people in north west england. Addiction Research \& Theory 1996; 4: 283-99.

42 Murgraff $V$, Parrott A, Bennett P. Risky single-occasion drinking amongst young people--definition, correlates, policy, and intervention: a broad overview of research findings. Alcohol Alcoholism 1999; 34: 3-14.

43 Baer JS, Kivlahan DR, Blume AW, McKnight P, Marlatt GA. Brief intervention for heavy-drinking college students: 4-year followup and natural history. Am J Public Health 2001; 91: 1310-16.

44 Wechsler $\mathrm{H}, \mathrm{Nelson}$ TF. What we have learned from the Harvard School of Public Health College Alcohol Study: focusing attention on college student alcohol consumption and the environmental conditions that promote it. J Stud Alcohol Drugs 2008; 69: 481-90.

45 Wechsler H, Nelson TF. Relationship between level of consumption and harms in assessing drink cut-points for alcohol research: Commentary on "Many college freshmen drink at levels far beyond the binge threshold" by White et al. Alcohol Clin Exp Res 2006; 30: 922-27.

46 Read JP, Beattie M, Chamberlain R, Merrill JE. Beyond the "Binge" threshold: heavy drinking patterns and their association with alcohol involvement indices in college students. Addict Behav 2008; 33: 225-34.

47 International Center for Alcohol Policies. ICAP blue book. Practical guides for alcohol policy and prevention approaches. [Citado 4 de diciembre de 2003]. Disponible en: http/www.icap. org/Portals/o/download/all_pdfs/blue_book/Module20_

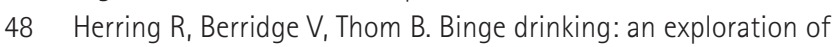
a confused concept. J Epidemiol Community Health 2008; 62: 476-79. 
49 McAlaney J, McMahon J. Establishing rates of binge drinking in the UK: anomalies in the data. Alcohol Alcoholism 2006; 41: 355-57.

50 National Institute on Alcohol Abuse and Alcoholism. NIAAA council aapproves definition of Binge Drinking. [Citado 15 de diciembre de 2004]. Disponible en: http//pubs.niaaa.nih.gov/ publications/Newsletter/winter2004/Newsletter_Number3.pdf.

51 Hustad JT, Carey KB. Using calculations to estimate blood alcohol concentrations for naturally occurring drinking episodes: a validity study. J Stud Alcohol 2005; 66: 130-38.

52 Kypri K, Langley J, Stephenson S. Episode-centred analysis of drinking to intoxication in university students. Alcohol Alcoholism 2005; 40: 447-52.

53 Cranford JA, McCabe SE, Boyd CJ. A new measure of binge drinking: prevalence and correlates in a probability sample of undergraduates. Alcohol Clin Ex Res 2006; 30: 1896-905.

54 Knight JR, Wechsler $H$, Kuo M, Seibring M, Weitzman ER, Schuckit MA. Alcohol abuse and dependence among U.S. college students. J Stud Alcohol 2002; 63: 263-270.

55 Vik PW, Tate SR, Carrello P. Detecting college binge drinkers using an extended time frame. Addict Behav 2000; 25: 607-612.

56 Calafat $A$, Juan $M$, Becoña $E$, Castillo $A$, Fernández $C$, Franco M, Pereiro $C_{1}$ Ros M. El consumo de alcohol en la lógica del botellón. Adicciones 2005; 17: 193-202.

57 Calafat A. El abuso de alcohol de los jóvenes en España. Adicciones 2007; 19: 217-224.

58 Farke W, Anderson P. El consumo concentrado de alcohol en Europa. Adicciones 2007; 19:333-340.

59 Crego A, Rodríguez Holguín S, Parada M, Mota N, Corral M, Cadaveira F. Binge Drinking affects attentional and visual working memory processing in young university students. Alcohol Clin Exp Res 2009; 33:1-10.

60 Goudriann AE, Grekin ER, Sher JK. Decision making and binge drinking: a longitudinal study. Alcohol Clin Exp Res 2007; 31: 928-38.

61 Hartley DE, Elsabagh S, File SE. Binge drinking and sex: effects on mood and cognitive function in healthy young volunteers. Pharmacol Biochem Behav 2004; 78: 611-619.

62 Davey JD. Determinants of binge drinking and alcohol use by young Australian women. J Child Adolesc Psychiatr Nurs 1997; 10: 7-16.

63 Engineer R, Philips A, Thompson J, Nicholls J. Drunk and disorderly: a qualitative study of binge drinking among 18 to 24 year old. London: Home Office Research Studies, 2003.

64 Guise JM, Gill JS. 'Binge drinking? It's good, it's harmless fun': a discourse analysis of accounts of female undergraduate drinking in Scotland. Health Educ Res 2007; 22: 895-906.
65 Richardson $\amalg$, Budd T. Young adults, alcohol, and crime and disorder. Criminal Behav Mental Health 2003; 13: 5-16.

66 Ariza C, Nebot M. Factors associated with problematic alcohol consumption in shoolchildren. J Adolesc Health 2000; 27: 42533.

67 Townshend JM, Duka T. Patterns of alcohol drinking in a population of young social drinkers: a comparison of questionnaire and diary measures. Alcohol Alcoholism 2002; 37: 187-92.

68 McCarty CA, Ebel BE, Garrison MM, DiGiuseppe DL, Christakis $D A$, Rivara FP. Continuity of binge and harmful drinking from late adolescence to early adulthood. Pediatrics 2004; 114: 714719 .

69 Daeppen JB, Anex F, Leutwyler J, Gammeter R, Darioli R, Pecoud A. Binge drinking in 19 year old men. Swiss Med Wkly 2005; 135: 179-183.

70 Guilamo-Ramos V, Jaccard J, Turrisi R, Johansson M, Parental and school correlates of binge drinking among middle school students. Am J Public Health 2005; 95: 894-99.

71 Nelson TF, Naimi TS, Brewer RD, Wechsler H. The state sets the rate: the relationship among state-specific college binge drinking, state binge drinking rates, and selected state alcohol control policies. Am J Public Health 2005; 95: 441-46.

72 D'Alessio M, Baiocco R, Laghi F. The problem of binge drinking among Italian university students: a preliminary investigation. Addict Behav 2006; 31: 2328-333.

73 Griffiths S, Lau JT, Chow JK, Lee SS, Kan PY, Lee S. Alcohol use among entrants to a Hong Kong University. Alcohol Alcoholism 2006; 41: 560-65.

74 Isralowitz R, Reznik A. Brief report: Binge drinking among highrisk male and female adolescents in Israel. J Adolesc 2006; 29: 845-49.

75 Xing Y, Ji C, Zhang L. Relationship of binge drinking and other health-compromising behaviors among urban adolescents in China. J Adolesc Health 2006; 39: 495-500.

76 Gill JS, Donaghy M, Guise J, Warner P. Descriptors and accounts of alcohol consumption: methodological issues piloted with female undergraduate drinkers in Scotland. Health Educ Res 2007; 22: 27-36.

77 Keller S, Maddock JE, Laforge RG, Velicer WF, Basler HD. Binge drinking and health behavior in medical students. Addict Behav 2007; 32: 505-15

78 Caamaño-Isorna $F_{1}$ Corral $M$, Parada $M$, Cadaveira F. Factors associated with risky consumption and heavy episodic drinking among spanish university students. J Stud Alcohol Drugs 2008; 69: 308-12. 
79 Page RM, Ihasz F, Hantiu I, Simonek J, Klarova R. Social normative perceptions of alcohol use and episodic heavy drinking among Central and Eastern European adolescents. Substance Use \&t Misuse 2008; 43: 361-73.

80 Kypri K, Paschal MJ, Langley J, Baxter J, Cashell-Smith M, Bourdeau B. Drinking and alcohol-related harm among New Zealand university students: findings from a natural web-based survey. Alcohol Clin Exp Res 2009; 33: 307-314 
